\title{
PARTIAL DIFFERENTIAL EQUATIONS ON SEMISIMPLE LIE GROUPS
}

\author{
KENNETH D. JOHNSON
}

\begin{abstract}
Suppose $G$ is a noncompact, connected, semisimple Lie group with finite center and $K$ is a maximal compact subgroup. Let $D$ be an Ad $K$ invariant element in the complexified enveloping algebra of $G$. The main result of this paper gives criterion for when the map $D: \mathscr{\delta}^{\prime}(G) \rightarrow \mathcal{E}^{\prime}(G)$ is injective, where $\mathscr{E}^{\prime}(G)$ is the space of compactly supported distributions on G.
\end{abstract}

1. Introduction. Let $G$ be a noncompact, connected, semisimple, Lie group with finite center. Fix $G=K A N$, an Iwasawa decomposition of $G$. That is, $K$ is a maximal compact subgroup of $G, A$ is a vector subgroup of $G$ with $\operatorname{Ad} A$ consisting of semisimple transformations and $A$ normalizes $N$, a simply connected nilpotent subgroup of $G$. Let $M$ be the centralizer of $A$ in $K$. We denote the Lie algebras of $G, K, M, A$, and $N$ by $\mathfrak{g}, \mathfrak{f}, \mathfrak{m}$, $\mathfrak{a}$, and $\mathfrak{n}$ respectively.

We use the notation $\mathscr{E}(G)=C^{\infty}(G)$ and $\mathscr{D}(G)=C_{0}^{\infty}(G)$ and denote their respective duals by $\mathcal{E}^{\prime}(G)$ and $\mathscr{Q}^{\prime}(G)$. Left and right translation by elements of $G$ induce linear maps on these spaces as follows.

For $x, g \in G$ and $f$ in either $\mathscr{D}(G)$ or $\mathcal{E}(G)$,

$$
L(g) f(x)=f\left(g^{-1} x\right), \quad R(g) f(x)=f(x g),
$$

and for $T$ in the dual of $f$,

$$
\langle L(g) T, f\rangle=\left\langle T, L\left(g^{-1}\right) f\right\rangle, \quad\langle R(g) T, f\rangle=\left\langle T, R\left(g^{-1}\right) f\right\rangle
$$

$(\langle$,$\rangle is complex bilinear).$

Let $\mathcal{E}(G: F)\left(\mathscr{D}^{\prime}(G: F)\right)$ denote the $C^{\infty}$ functions (distributions) on $G$ which are left and right $K$-finite and set

$$
\mathcal{E}^{\prime}(G: F)=\mathcal{E}^{\prime}(G) \cap \mathscr{D}^{\prime}(G: F), \quad \mathscr{D}(G: F)=\mathcal{E}(G: F) \cap \mathscr{D}(G) .
$$

Let $U(\mathfrak{g})$ denote the universal enveloping algebra of the complexification of $\mathfrak{g}$, let $U(\mathfrak{g})^{\mathfrak{f}}$ be the centralizer of $\mathfrak{f}$ in $U(\mathfrak{g})$ and $\mathscr{Z}$ the center of $U(\mathrm{~g})$. The main purpose of this paper is to give a criterion (Lemma 3.1) for when $D(\mathscr{E}(G)$ ) is dense in $\mathscr{E}(G)$ for $D \in U(\mathfrak{g})^{\mathfrak{f}}$ and to apply this criterion to elements of $\mathcal{Z}$. In this paper we only prove that this criterion is sufficient, but in a subsequent paper [6], we show that this criterion is necessary for linear noncompact semisimple Lie groups.

Finally, we apply our techniques to the study of invariant differential

Received by the editors December 26, 1974.

AMS (MOS) subject classifications (1970). Primary 22E30; Secondary 43A30, 35A22. 
operators on homogeneous vector bundles. In this paper we do not need to prove an analog of the classical Paley-Wiener theorem but rest our main techniques on Harish-Chandra's characterization of cusp forms and his Fourier expansions of $C^{\infty}$-functions on $G$.

2. Principal series and the Fourier transform. We first recall the definition of the principal series of $G$. Let $\omega: M \rightarrow G l(H)$ be an irreducible unitary representation of $M$ and let $\nu \in \mathrm{a}_{\mathrm{c}}^{*} \cdot \omega$ and $\nu$ define a representation $\nabla_{\omega, \nu}$ of the group $M A N=P_{0}$ on $H$ by setting $\nabla_{\omega, \nu}($ man $)=e^{(i v+\rho)(\log a)} \omega(m)(m \in M, a$ $\in A, n \in N)$ where, for $H \in \mathfrak{a}, 2 \rho(H)=\operatorname{tr}$ ad $\left.H\right|_{\mathfrak{n}}$. Now let $H^{\omega, \nu}$ be the set of all measurable functions $f: G \rightarrow H$ such that:

(1) $f(g p)=\nabla_{\omega, \nu}(p)^{-1} f(g)\left(g \in G, p \in P_{0}\right)$; and

(2) $\int_{K}\|f(k)\|^{2} d k=\|f\|^{2}<\infty$.

Observe that we have an inner product on $H^{\omega, \nu},(u, v)=\int_{K}(u(k), v(k)) d k$ $\left(u, v \in H^{\omega, \nu}\right)$, and this turns $H^{\omega, \nu}$ into a Hilbert space. Now left translation induces a representation $\Pi_{\omega, \nu}$ of $G$ on $H^{\omega, \nu}$. Let $X^{\omega, \nu}$ denote the set of all $K$ finite vectors of $H^{\omega, \nu}$ and observe that as a $K$-module, $X^{\omega, \nu}$ is isomorphic to the space

$$
\begin{array}{r}
X(\omega)=\left\{u: K \rightarrow H \mid u \text { is left } K \text {-finite and } u(k m)=\omega(m)^{-1} u(k)\right. \\
\text { for all } k \in K, m \in M\} .
\end{array}
$$

. We will abuse notation throughout and identify $X^{\omega, \nu}$ with $X(\omega)$ and any distinction will be carried by the term $\Pi_{\omega, v}$. Observe now that $\Pi_{\omega, \nu}$ induces a representation of the algebra $U(\mathfrak{g})$ on $X(\omega)$.

If $f \in D(G)$ we define, as usual,

$$
\Pi_{\omega, \nu}(f)=\int_{G} f(x) \Pi_{\omega, \nu}(x) d x .
$$

If $T \in \mathcal{E}^{\prime}(G)$ we may attempt to extend the above definition by setting $\left(\Pi_{\omega, \nu}(T) u, v\right)=\langle T, h\rangle$, where $u, v \in H^{\omega, \nu}$ and $\left(\Pi_{\omega, \nu}(x) u, v\right)=h(x)$. However, as $h(x)$ is not necessarily $C^{\infty}$, we see that for a general such $T, \Pi_{\omega, \nu}(T)$ is only densely defined. Suppose now that $T \in \mathcal{E}^{\prime}(G: F)$ and define, for $u, v \in X(\omega)$, $\left(\Pi_{\omega, \nu}(T) u, v\right)$ as above. Note that $\Pi_{\omega, \nu}(T)$ restricted to $X(\omega)$ is well defined and that $\left(\Pi_{\omega, \nu}(T) u, v\right)=0$ if $u, v$ are not in a minimal $\Pi_{\omega, \nu}(T)$ invariant space, which we denote as $X(\omega, T)$. As the closure of $X(\omega, T)$ in $H^{\omega, \nu}$ is $X(\omega, T)$, $\Pi_{\omega, \nu}(T)$ extends to a linear operator on $H^{\omega, \nu}$.

LEMMA 2.1. Fixing $\omega \in \hat{M}$ we have that the map $\mathrm{a}_{c}^{*} \rightarrow \operatorname{End} X(\omega, T)$ $\subseteq$ End $H^{\omega, \nu}$, given by $\nu \rightarrow \Pi_{\omega, \nu}(T)$, is holomorphic.

Proof. Now $\Pi_{\omega, \nu}(T)$ has finite rank, and it suffices to show that for a fixed $u, v \in X(\omega)$ the map $\nu \rightarrow\left(\Pi_{\omega, \nu}(T) u, v\right)$ is holomorphic. Now set $f_{\nu}(x)$ $=\left(\Pi_{\omega, \nu}(x) u, v\right)$, and we have that $\left(\Pi_{\omega, \nu}(T) u, v\right)=\left\langle T, f_{\nu}\right\rangle$, where

$$
f_{\nu}(x)=\int_{K} \exp \left\{-(i \nu+\rho) H\left(\rho^{-1} k\right)\right\}\left(u\left(K\left(x^{-1} k\right), v(k)\right)\right) d k,
$$

where for $g \in G, \quad g=K(g) \exp H(g) n(g)(K(g) \in K, H(g) \in a, n(g)$ $\in N)$. As $T \in \mathcal{E}^{\prime}(G)$ we have that there exists a compact set $V \subseteq G$ such that 
supp $T \subset V$ and $K V K=V$. Now there exist $D_{1}, \ldots, D_{n} \in U(\mathrm{~g})$ and $C>0$ such that for $f \in \mathscr{D}(G)$,

$$
|\langle T, f\rangle| \leq C \sum_{i=1}^{n} \sup _{x \in V}\left|D_{i} f(x)\right| .
$$

For $e \in \mathrm{a}_{\mathrm{c}}^{*}$ and for $W$ a compact set with int $W \supset V$, we see that $1 / \xi\left(f_{v+\xi e}(x)-f_{\nu}(x)\right)$ converges uniformly on $W$ as $\xi \rightarrow 0$ to

$$
F_{\nu}(x)=\int_{K}(-i) e\left(H\left(x^{-1} k\right)\right) e^{-(i \nu+\rho)\left(H\left(x^{-1} k\right)\right)}\left(U\left(K\left(x^{-1} k\right)\right), v(k)\right) d k
$$

and if $D \in U(\mathfrak{g}), 1 / \xi\left(D f_{v+\xi e}(x)-D f_{v}(x)\right)$ converges uniformly to $D F_{v}(x)$ on $W$ by the Leibniz formula. Thus $\left\langle T, 1 / \xi\left(f_{v+\xi e}-f_{v}\right)\right\rangle$ converges to $\left\langle T, F_{\nu}\right\rangle$ as $\xi \rightarrow 0$ and, hence, $\nu \rightarrow\left(\Pi_{\omega, \nu}(T) u, v\right)$ is holomorphic.

The map $\nu \rightarrow \Pi_{\omega, \nu}(T)$ also satisfies growth conditions which we will not examine here.

Lemma 2.2. Suppose $T \in \mathcal{E}^{\prime}(G: F)$ and $\Pi_{\omega, \nu}(T)=0$ for all $\omega \in \hat{M}$ and all $\nu \in \mathrm{a}_{\mathbf{c}}^{*}$. Then $T=0$.

Proof. Let $g \in D(G)$ and consider the function $g_{*} T \in \mathscr{D}(G)$ where $g_{*} T(x)=\left\langle T, L_{x^{-1}} g\right\rangle$ with $\tilde{g}(y)=g\left(y^{-1}\right)$. An elementary calculation then yields $\Pi_{\omega, \nu}\left(g_{*} T\right)=\Pi_{\omega, \nu}(g) \Pi_{\omega, \nu}(T)$. Thus, setting $f=g_{*} T$, we have that $\Pi_{\omega, \nu}(f) \stackrel{=}{=} 0$ for all $\omega \stackrel{M}{\in}$ and all $\nu \in a_{\mathbf{c}}^{*}$. We now examine $\Pi_{\omega, \nu}(f)$ more closely.

Let $h: K \rightarrow H$ be a continuous function such that $h(k m)=\omega(m)^{-1} h(k)$ for $k \in K, m \in M$. Then, for any $\nu \in \mathrm{a}_{\mathbf{c}}^{*}, h$ extends to a function in $H^{\omega, \nu}$. Now

$$
\Pi_{\omega, \nu}(f)=\int_{G} f(x) h\left(x^{-1} k\right) d k=\int_{K} K_{f, \nu}\left(k, k^{\prime}\right) h\left(k^{\prime}\right) d k^{\prime}
$$

where

$$
K_{f, \nu}\left(k, k^{\prime}\right)=\int_{A} d a \int_{N} d n \int_{M} d m \omega(m) f\left(k m a n k^{\prime-1}\right) e^{-(i v+\rho)(\log a)} .
$$

As $f \in \mathscr{D}(G)$, we see that $K_{f, \nu}\left(k, k^{\prime}\right)$ is continuous, and since $\Pi_{\omega, \nu}(f)=0$ for all $\omega \in \hat{M}$, we know that $K_{f, \nu}=0$ for all $\nu \in a_{\mathbf{c}}^{*}$. From the Plancherel formula for $\mathbf{R}^{n}$ we see that

$$
\int_{N} d n f\left(k a n k^{\prime-1}\right)=0 \quad\left(k, k^{\prime} \in K, a \in A\right) .
$$

Now let $U$ be the unipotent radical of a minimal parabolic subgroup of $G$ and consider the function

$$
F(g)=\int_{U} f(g u) d u .
$$

As is well known there is a $k^{\prime} \in K$ such that $U=k^{\prime} N k^{\prime-1}$. Thus

$$
F(g)=\int_{N} f\left(g k^{\prime} n k^{\prime-1}\right) d n .
$$

Now writing $g k^{\prime}=k a n_{1}\left(k \in K, a \in A, n_{1} \in N\right)$ we see that 


$$
F(g)=\int_{N} f\left(\text { kank }^{\prime-1}\right) d n=0
$$

for any $g \in G$. We now show that $f=0$.

Let $P_{1}$ be a parabolic subgroup of $G$ which is minimal with respect to the property that

$$
f_{P_{1}}(g)=\int_{U_{1}} f(g u) d u \not \equiv 0,
$$

where $U_{1}$ is the unipotent radical of $P_{1}$. Since $f$ is the (possibly infinite) sum of functions in $\mathscr{D}(G: F)$ (see Harish-Chandra [3]), to show that $f=0$ it suffices to prove $f=0$ under the assumption that $f \in \mathscr{D}(G: F)$. Now $P_{1}=M_{1} U_{1}$, where $M_{1}$ is reductive, and thus we have that $f_{P_{1}}$ is a cusp form on $M_{1}$ with compact support. From Harish-Chandra [2] we see that $f_{P_{1}}=0$ since $P_{1}$ is not conjugate to $P_{0}$, which is a contradiction. Therefore, $f=0$.

As $g_{*} T=0$ for any $g \in \mathscr{D}(G)$, we have that $T=0$.

Lemma 2.3. For $D \in U(\mathfrak{g})^{\mathfrak{f}}, D: \mathcal{E}^{\prime}(G) \rightarrow \mathcal{E}^{\prime}(G)$ is injective if and only if $D: \mathcal{E}^{\prime}(G: F) \rightarrow \mathcal{E}^{\prime}(G: F)$ is injective.

Proof. The only if part is obvious. Suppose now that $D: \mathcal{E}^{\prime}(G: F)$ $\rightarrow \mathcal{E}^{\prime}(G: F)$ is injective. Let $f \in \mathcal{E}(G)$ and observe from Harish-Chandra [3] that

$$
f=\sum_{\nabla, \tau \in \hat{K}} \chi_{\nabla}{ }^{*}{ }_{K} f{ }^{*} \chi_{\tau} \quad \text { where } \chi_{\nabla}(k)=(\operatorname{deg} \nabla) \operatorname{tr} \nabla(k),
$$

and this series converges in $\mathcal{E}(G)$, and for $T \in \mathcal{E}^{\prime}(G)$ we have

$$
\langle T, f\rangle=\sum_{\nabla, \tau \in \hat{K}}\left\langle T, \chi_{\nabla}{ }^{*} K{ }^{*}{ }_{K} \chi_{\tau}\right\rangle .
$$

Next let $0 \neq T \in \mathcal{E}^{\prime}(G)$ be such that $D T=0$. As $D T=0$, we have that $\left\langle D T, \chi_{\nabla}{ }^{*} K{ }^{*}{ }_{K} \chi_{\tau}\right\rangle=0$ for all $\nabla, \tau \in \hat{K}$. Fixing $\nabla_{0}, \tau_{0} \in \hat{K}$, we suppose that $\left\langle T, \chi_{\nabla_{0}}{ }^{*} K{ }^{*} K \chi_{\tau_{0}}\right\rangle \neq 0$. Now define $T_{0} \in \mathcal{E}^{\prime}(G)$ by setting $\left\langle T_{0}, f\right\rangle=\left\langle T, \chi_{\nabla_{0}}\right.$ ${ }^{*} K{ }^{*}{ }_{K} \chi_{\tau_{0}}>$ for $f \in \varepsilon(G)$. Now $T_{0} \in \mathcal{E}^{\prime}(G: F)$ and $D T_{0}=0$ as $D \in U(\mathfrak{g})^{t^{0}}$. Thus, $T_{0}=0$, which is a contradiction. Hence, $D: \mathcal{E}^{\prime}(G) \rightarrow \mathcal{E}^{\prime}(G)$ is injective.

3. Injectivity criterion. Suppose $D \in U(\mathfrak{g})^{\mathfrak{f}}$. In this section, we give a criterion for the operator $D: \mathcal{E}^{\prime}(G) \rightarrow \mathcal{E}^{\prime}(G)$ to be injective and derive some consequences of this criterion for operators in $Z$.

LEMMA 3.1 (InJectivity CRITERION). Suppose $D \in U(\mathfrak{g})^{\mathfrak{f}}$. Then $D: \mathcal{E}^{\prime}(G)$ $\rightarrow \mathcal{E}^{\prime}(G)$ is injective if for no $\omega \in \hat{M}$ is there a finite dimensional space $V \subseteq X(\omega)$ such that $\Pi_{\omega, \nu}(D): V \rightarrow V$ and det $\left.\Pi_{\omega, \nu}(D)\right|_{V}=0$ for all $\nu$.

Proof. Let $T \in \mathcal{E}^{\prime}(G: F)$ and fix $\omega \in \hat{M}$ such that $\nu \rightarrow \Pi_{\omega, \nu}(T) \not \equiv 0$. Now

$$
\Pi_{\omega, \nu}(T): X(\omega, T) \rightarrow X(\omega, T),
$$

and as $D \in U(\mathfrak{g})^{\mathfrak{t}}$, there is a $V \supseteq X(\omega, T)$ such that $\Pi_{\omega \nu}(D): V \rightarrow V$. Now as $\nu \rightarrow \Pi_{\omega, \nu}(D T)=\Pi_{\omega, \nu}(D) \Pi_{\omega, \nu}(T)$ is analytic and det $\left.\Pi_{\omega, \nu}(D)\right|_{\nu} \not \equiv 0$, we have that $\nu \rightarrow \Pi_{\omega, \nu}(D) \Pi_{\omega, \nu}(T) \not \equiv 0$. 
LEMMA 3.2. If $D \in U(\mathfrak{g})^{\mathfrak{f}}$ satisfies the injectivity criterion of Lemma 3.1, then so does $D^{*}$, the adjoint of $D$.

Proof. Let $\rho^{*}: U(\mathfrak{g}) \rightarrow U(\mathfrak{g})$ be the linear map such that

$$
\rho^{*}\left(X_{1} \cdots X_{r}\right)=(-1)^{r} X_{r} \cdots X_{1} \quad\left(X_{i} \in g_{\mathbf{c}}\right) .
$$

Then $D^{*}=\rho^{*}(D)$, and our result follows from the fact that, for $u, v \in X(\omega)$, $\left(\Pi_{\omega, \nu}(D) u, v\right)=\left(u, \Pi_{\omega, \bar{\nu}}\left(D^{*}\right) v\right)$.

Set $R(X) f(g)=\left.(d / d t) f(g \exp t X)\right|_{t=0}$ and extend $R$ to a representation of $U(g)$ on $\mathcal{E}(G), \mathscr{D}(G), \mathcal{E}^{\prime}(G)$ and $\mathscr{D}^{\prime}(G)$. Then we easily obtain for $\omega \in \hat{M}$ and $\nu \in a_{c}^{*}$ that, for $T \in \mathcal{E}^{\prime}(G), \Pi_{\omega, \nu}(R(D) T)=\Pi_{\omega, \nu}(T) \Pi_{\omega, \nu}\left(\rho^{*}(D)\right)$.

We now easily obtain, from Lemma 3.2,

LEMMA 3.3. If $D \in U(\mathfrak{g})^{\mathfrak{t}}$ satisfies the injectivity criterion of Lemma 3.1, the linear map $R(D): \mathcal{E}^{\prime}(G) \rightarrow \mathcal{E}^{\prime}(G)$ is injective.

Suppose now that $D \in \mathcal{Z}$. Then, as is well known, there is a unique $D_{0} \in \mathscr{Z}(\mathrm{m} \oplus \mathfrak{a})$, the center of $U(\mathrm{~m} \oplus \mathrm{a})$, such that $D-D_{0} \in \mathfrak{n} U(G)$ and the map $D \rightarrow D_{0}$ is an injective ring homomorphism of $\mathscr{Z}$ into $\mathscr{Z}(\mathfrak{m} \oplus \mathfrak{a})$. Now as $D \in \mathscr{Z}$, the map $\nu \rightarrow \Pi_{\omega, \nu}(D)$ is an analytic map into $\mathbf{C}$ and $\Pi_{\omega, \nu}(D)$ $=\nabla_{\omega, \nu}\left(\rho^{*}\left(D_{\nu}\right)\right)$. Letting $H_{1}, \ldots, H_{l}$ be a basis for a we see that

$$
D_{0}=\sum_{j=1 \leqslant i_{1} \leqslant \cdots \leqslant i_{j} \leqslant l}^{r} z\left(i_{1}, \ldots, i_{j}\right) H_{i_{1}} \cdots H_{i_{j}}
$$

with each $z\left(i_{1}, \ldots, i_{j}\right)$ in the center of $U(\mathrm{~m})$. Then

$$
\begin{aligned}
\nabla_{\omega, \nu}\left(\rho^{*}\left(D_{0}\right)\right)=\sum_{j=1}^{r} \sum_{1 \leqslant i_{1} \leqslant \cdots \leqslant i_{j} \leqslant l}(-1)^{j} \omega\left(\rho^{*}\left(z\left(i_{1}, \ldots, i_{j}\right)\right)\right) \\
\cdot(\rho+i \nu)\left(H_{j}\right) \cdots(\rho+i \nu)\left(H_{i_{j}}\right)
\end{aligned}
$$

and thus we see that $D$ satisfies the injectivity criterion of Lemma 3.1 if and only if for no $\omega \in \hat{M}$ do all $\left(\rho^{*}\left(z\left(i_{1}, \ldots, i_{j}\right)\right)\right)=0$.

The following result is now obvious.

THEOREM 3.1. If $G$ is a split semisimple Lie group and $0 \neq D \in \mathcal{Z}$, then $D: \mathcal{E}^{\prime}(G) \rightarrow \mathcal{E}^{\prime}(G)$ is injective.

4. Homogeneous vector bundles on symmetric spaces. We conclude by applying our techniques to homogeneous vector bundles over $G / K$.

Let $\tau: K \rightarrow G l(V)$ be an irreducible unitary representation of $K$ and suppose that $\omega=\left.\tau\right|_{M}$ is irreducible. (We include the case where $\tau$ is trivial.) $\tau$ then determines a homogeneous vector bundle $G \times{ }_{\tau} V$ over $G / K$ and we identify the cross sections of this vector bundle with functions $f: G \rightarrow V$ such that:

$$
f(g k)=\tau(k)^{-1} f(g) \quad(g \in G, k \in K) .
$$

Let $\mathcal{E}(G: \tau)(\mathscr{D}(G: \tau))$, denote the $C^{\infty}$-cross sections (with compact support). Let $\mathcal{E}^{\prime}(G: \tau)\left(\mathscr{D}^{\prime}(G: \tau)\right)$ be the dual of $\mathcal{E}(G: \tau)(\mathscr{D}(G: \tau))$. Extend $T$ $\in \mathcal{E}^{\prime}(G: \tau)$ to an operator on all $C^{\infty}$-functions $f: G \rightarrow V$ by setting 


$$
\langle T, f\rangle=\left\langle T, f{ }^{*} \chi_{\tau^{*}}\right\rangle
$$

where $\tau^{*}(k)=\left(\tau(k)^{-1}\right)^{t}$, and extend the elements of $\mathscr{D}^{\prime}(G: \tau)$ accordingly. Let $\mathcal{E}(G: \tau: F), \mathscr{D}(G: \tau: F), \mathcal{E}^{\prime}(G: \tau: F)$, and $\mathscr{D}^{\prime}(G: \tau: F)$ denote, respectively, the elements of $\mathcal{E}(G: \tau), \mathscr{D}(G: \tau), \mathcal{E}^{\prime}(G: \tau)$, and $\mathscr{D}^{\prime}(G: \tau)$ which are left $K$-finite.

If $D \in U(\mathfrak{g})^{\mathfrak{f}}$ we see that $R(D)$ maps each of the above eight spaces into itself. In this section, we show that if $R(D) \not \equiv 0$ on $\mathcal{E}^{\prime}(G: \tau), R(D): \mathcal{E}^{\prime}(G: \tau)$ $\rightarrow \mathcal{E}^{\prime}(G: \tau)$ is injective.

Observe that we have the following.

LEMMA 4.1. If $f \in \mathcal{E}(G: \tau), f{ }^{*} \chi_{\tau^{*}}=f$.

LEMMA 4.2. If $f \in \mathscr{D}(G: \tau)$ and $l \in V^{*}$, let $l(f): G \rightarrow \mathbf{C}$ be the function $l(f(x))=(l, f(x))$. Then for $g \in \hat{M}$ and $\nu \in a_{\mathbf{c}}^{*}, \Pi_{\phi, \nu}(l(f))=0$ unless $\phi$ $=\omega^{*}$. Moreover, if $u \in X\left(\omega^{*}\right), \nabla \in \hat{K}$ and $\chi_{\nabla}{ }^{*} K u \stackrel{\phi}{=} u$, then $\Pi_{\omega^{*}, v}(l(f)) u$ $=0$ unless $\nabla=\tau^{*}$.

Proof. Let $u \in H^{\phi, \nu}$. Then

$$
\left(\Pi_{\phi, \nu}(l(f)) u\right)(k)=\int_{G}(l, f(x))\left(\Pi_{\phi, \nu}(x) u\right)(k) d x .
$$

As $l(f)=l(f){ }^{*} \chi_{T}$, we see that $\Pi_{\phi, \nu}(l(f)) u(k)=\Pi_{\phi, \nu}(l(f))\left(\chi_{\tau^{*}}{ }^{*}{ }_{K} u\right)(k)$. Thus $\Pi_{\phi, \nu}(l(f)) \neq 0$ only if there is a $u \in X(\phi)$ for which $\chi_{\tau^{*}}{ }^{*} K u=u$. This yields our result.

Observe by Frobenius reciprocity that the multiplicity of $\tau^{*}$ in $X\left(\omega^{*}\right)$ is 1 .

Lemma 4.3. Let $T \in \mathcal{E}^{\prime}(G: \tau: F)$ and $e \in V$. Let $T \otimes e \in \mathcal{E}^{\prime}(G: F)$ be defined by $\langle T \otimes e, f\rangle=\langle T, f e\rangle$. Then for $g \in \hat{M}$ and $\nu \in a_{c}^{*}, \Pi_{\phi, \nu}(T \otimes e)=0$ unless $\phi=\omega$. Moreover, if $u \in X(\omega), \nabla \in K$ and $X_{\nabla}{ }^{*} K u=u$, then

$$
\Pi_{\omega, \nu}(T \otimes e) u=0
$$

unless $\nabla=\tau$.

Proof. Let $f \in \mathscr{D}(G)$ and consider $f_{*} T$ in place of $T$. The proof for $f_{*} T$ is the same as the proof of Lemma 4.2.

LEMMA 4.4. Let $T \in \mathcal{E}^{\prime}(G: \tau: F)$ be $\neq 0, D \in U(\mathrm{~g})^{\mathfrak{t}}$, and $V^{0}=\{f$ $\left.\in X(\omega): \chi_{\tau}{ }_{K} f=f\right\}$. Then $\left.\Pi_{\omega, \nu}\left(\rho^{*}(D)\right)\right|_{V^{0}}$ is a scalar operator and $\nu$ $\left.\rightarrow \Pi_{\omega, \nu}\left(\rho^{*}(D)\right)\right|_{V^{0}}$ is a polynomial. Furthermore, $R(D) T=0$ if and only if $\left.\nu \rightarrow \Pi_{\omega, \nu}\left(\rho^{*}(D)\right)\right|_{V^{0}} \equiv 0$.

Proof. As $(\tau: \omega)=1, \tau$ occurs once and only once in $X(\omega)$. Hence, $\left.\Pi_{\omega, \nu}\left(\rho^{*}(D)\right)\right|_{V^{0}}$, since it commutes with $K$, is a scalar operator. It is well known that $\left.\nu \rightarrow \Pi_{\omega, \nu}\left(\rho^{*}(D)\right)\right|_{V^{0}}$ is a polynomial map. As $\Pi_{\omega, \nu}(R(D) T)$ $=\Pi_{\omega, \nu}(T) \Pi_{\omega, \nu}\left(\rho^{*}(D)\right)$, our result follows from Lemmas 2.2 and 4.3.

THEOREM 4.1. Let $D \in U(\mathfrak{g})^{\mathfrak{f}}$ and suppose that the map $R(D): \mathcal{E}^{\prime}(G: \tau)$ $\rightarrow \mathcal{E}^{\prime}(G: \tau)$ is $\not \equiv 0$. Then $R(D): \mathcal{E}^{\prime}(G: \tau) \rightarrow \mathcal{E}^{\prime}(G: \tau)$ is injective.

Proof. If $R(D) T=0$ for $0 \neq T \in \mathcal{E}^{\prime}(G: \tau)$, then $\left.\nu \rightarrow \Pi_{\omega, \nu}\left(\rho^{*}(D)\right)\right|_{V^{0}}=0$ and, hence, $R(D) \mathcal{E}^{\prime}(G: \tau)=0$ by Lemmas $2.2,4.3$ and 4.4. Hence, $R(D)$ : $\mathcal{E}^{\prime}(G: \tau) \rightarrow \mathcal{E}^{\prime}(G: \tau)$ is injective. 
Remark. For $\tau$ trivial this result was proved by S. Helgason.

\section{BIBLIOGRAPHY}

1. Harish-Chandra, Spherical functions on a semisimple Lie group. I, Amer. J. Math. 80 (1958), 241-310. MR 20 \#925.

2. - Spherical functions on a semisimple Lie group. II, Amer. J. Math. 80 (1958), 553613. MR 21 \#2.

3. —_, Discrete series for semisimple Lie groups. II, Acta Math. 116 (1966), 1-111. MR 36 \#2745.

4. _- On the theory of the Eisenstein integral, Lecture Notes in Math., vol. 266, SpringerVerlag, Berlin and New York, 1972, pp. 123-149.

5. S. Helgason, The surjectivity of invariant differential operators on symmetric spaces. I, Ann. of Math. (2) 98 (1973), $451-479$.

6. K. Johnson, Differential equations and an analog of the Paley-Wiener theorem for linear semisimple Lie groups, Nagoya Math. J. (submitted).

Department of Mathematics, Rutgers University, New Brunswick, New Jersey 08903 